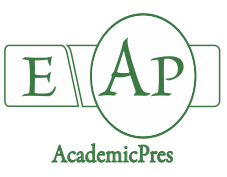

Yao Z et al. (2020)

Notulae Botanicae Horti Agrobotanici Cluj-Napoca 48(1):14-23

DOI: $10.15835 /$ nbha48111762

Research article

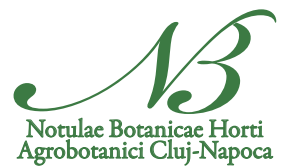

\title{
The chloroplast localization of protease mediated by the potato rbcS signal peptide and its improvement for construction of photorespiratory bypasses
}

\author{
Zhen $\mathrm{YAO}^{1 *}$, Boran $\mathrm{SHEN}^{2}$, Xiulan $\mathrm{YANG}^{3}$, \\ Minhui LONG ${ }^{4}$ \\ ${ }^{l}$ Yangtze University, College of Horticulture and Gardening, Jingzhou, 434025, \\ China; cjdxyaozhen@aliyun.com ("correspondingauthor) \\ ${ }^{2}$ South China Agricultural University, Laboratory of Molecular Plant Physiology, College of Life Sciences, Guangzhou, 510640, \\ China;185880915@qq.com \\ ${ }^{3}$ Yangtze University, Department of Medicine,Jingzhou, 434025, China; 406490115@qq.com \\ ${ }^{4}$ South China Normal University, College of Life science, Guangzhou, 510631, China; 490015018@qq.com
}

\begin{abstract}
Location of the proteases would affect on protease stability and photorespiratory bypass pathway, while it is unsolved. Potato rbcS signal peptide was analyzed and constructed into the protease for study of their localization site. The tartronate semialdehyde reductase (EcTSR) proteins could be accurately and efficiently located in chloroplast only when this signal peptide was extended to 80 amino acids. The signal peptide would help malate synthase (CmMS) locate to the surface of chloroplast, to form granules on the outer membrane of chloroplast. The whole spectrum scanning showed that these proteins could enter chloroplast. A signal peptide named PCS1 (Peptide of self-cleavage site 1) carrying a self-cleavage site was designed, and sixteen amino acids from the blue pigment precursor protein of chloroplast positioning signal of Silene pratensis were added to the C-terminal of PCS1. Transient expression, Western blot analysis and full-spectrum scanning showed that PCS1 could locate the EcTSR to the chloroplast, after the removal of the signal peptide.
\end{abstract}

Keywords: chloroplast localization; chloroplast signal peptide; photorespiratory bypass

\section{Introduction}

Photorespiration may result in a 25\% loss of photosynthates in C3 plants under normal conditions, the value will be even higher if stress conditions, such as drought, heat and high light, are encountered by the plant (Peterhansel and Maurino, 2011). In 2007, bacterial glycolic acid pathways were introduced into chloroplasts to construct the first photorespiration pathway called the Kebeish pathway (Kebeish et al., 2007). Subsequently, glycolate oxidase and malate synthase were located in chloroplast to build the second branch (Maier et al., 2012). By these ways, $75 \%$ of glycolic acid produced by photorespiration is transformed into the Calvin cycle. 
The photorespiration bypasses have adverse effects on $\mathrm{H}_{2} \mathrm{O}_{2}$ signaling (Rojas and Mysore, 2012; Zhang et al., 2016), amino acid metabolism and carbon production in plants (Dalal et al., 2015; Novitskaya et al., 2002). Some studies suggested that the CO2 conductance of chloroplast membrane is a key factor in the effectiveness of photorespiration (Xin et al., 2015). Despite these problems, these pathways have attractive prospects for increasing biomass and reducing $\mathrm{CO} 2$ deficiency and excess energy damage in stresses such as high temperature and drought. In 2015, Dalal achieved Kebeish branch on Camelina sativa to promote its growth and seed yield (Dalal et al., 2015). In 2019, the fourth bypass was successfully established in rice chloroplasts using a multi-gene assembly and transformation system (Shen et al., 2019).

The first kind of enzymes which were used to construct chloroplast photorespiration metabolic pathway was prokaryotic enzymes, including bacterial GLC (glycolate dehydrogenase), GCL (acetaldehyde polymerase), TSR (hydroxymalonate semi-aldehyde reductase), and CAT (catalase), all of them don't contain subcellular localization signal. The second kind of enzymes are eukaryotic enzymes, including GLO (glycolate oxidase) and MS (malic acid synthase), them are located in peroxidase and glyoxylate circulator respectively. These two types of enzymes, which are required for the construction of photorespiration metabolic pathways, were introduced into chloroplasts via chloroplast localization signal peptides. It is generally believed that chloroplast signal peptide is the strongest signal peptide with the ability of subcellular localization.

Chloroplast localization signal peptides usually contain a large number of hydroxyl amino acid residues (e.g. serine, threonine, proline) and are lack of acidic amino acid residues such as Aspartic acid and Glutamic acid (von Heijne et al., 1989). In addition, these signal peptides tend to form hydrophobic alpha-helix structures (Bruce, 2000). The length and primary structure of signal peptides are also diverse (Bruce, 2001). The rbcS chloroplast localization signal peptides of potato were first identified in 1988 (Wolter et al., 1988), and have been used for research of Nolke and Houdelet (2014).

Some failure example of location via chloroplast signal peptide would happen, for example, Lubben found that Brom Mosaic virus envelope protein was located on the chloroplast by pea rbcS signal peptide, and its efficiency was only 2.3\% (Lubben et al., 1989). Byeon found that the rice n-acetyltransferase signal peptide could not locate n-acetyltransferase derived from sheep into the rice chloroplast (Byeon $e t$ al., 2014).

As an important food crop, the construction of photorespiration metabolic pathway has great potential to improve potato yield. Although it has been reported that enzymes related to photorespiration metabolic pathways e.g., EcGCL, EcTSR, EcCAT, CmMS, and OsGLO3 can be imported into rice or Arabidopsis chloroplast by rbcS signal peptides (Kebeish et al., 2007; Maier et al., 2012; Shen et al., 2019), these enzymes were never tested in potato. In early study, we attempted to introduce enzymes, which were constructed for the photorespiration metabolic pathway, into the potato chloroplast through the chloroplast localization signal peptide. We found that some genes were highly expressed at the mRNA level but the protein level was low. If a key enzyme for metabolic pathway cannot complete its localization in chloroplast or has low efficiency of transportation and cannot obtain its appropriate enzyme activity in the chloroplast, the accumulation of intermediate products would be harmful to the chloroplast.

So, we analyzed the chloroplast localization of these proteases required for the construction of photorespiration pathways. We designed two cutting sites that were consisted of 20 amino acid residues respectively, and these cutting sites was separately added into the C-terminal of the chloroplast localization signal peptides of the potato $\mathrm{rbcS}$ gene to construct new signal peptides, and respectively named PCS1 (Peptide of self-cleavage site 1) and PCS2 (Peptide of self-cleavage site 2). Among them, PCS1 contains 16 amino acid residues from the plasmid precursor protein (P07030) of Silene pratensis, and its sequence is TVKVAVATPRMSIKAS (Richter and Lamppa, 1998). PCS2 contains 17 amino acid residues from the rbcS protein of Oryza sativa. Western blot analysis and full-spectrum scanning showed that PCS1 could locate protease to the chloroplast, after the removal of the signal peptide. PCS1 has potential applications in the construction of photorespiration metabolic pathways and chloroplast metabolic engineering. 


\section{Materials and Methods}

Reform plasmid $p B 1121$ and constructs the tobacco instantaneous expression vector

Using SmaI and SacI to remove GUS gene from original vector pBI121 gi19569229, replace with amplification of eGFP gene gi1721719622 construct carrier pBI-eGFP. Connected to target genes between 35 s promoter and eGFP gene, the target gene expression and eGFP genes constitute a box, expression of target genes and eGFP fusion protein.

Building of vectors for protein expression mediated by rbcS chloroplast localization signal peptide (58AA)

Amplified potato $\mathrm{rbcS}$ chloroplast localization signal peptide (StTP-58AA gi21562 174bp) sequence and target gene EcGCL, EcTSR, EcCAT (gi49175990), CmMS (hm755991), and OsGLO3 (nc029259). By connecting the pBI-eGFP, pStTP-58AA-EcTSR-eGFP, pStTP-58AA-EcCAT-eGFP, pStTP-58AAOsGLO3-eGFP, pStTP-58AA-CmMS-eGFP, pStTP-58AA-EcGCL-eGFP were constructed. Details of plasmid structure is presented in Figure 1.

Building of vectors for protein expression mediated by extended $r b c S$ chloroplast localization signal peptide prolongs

The length of 80 amino acids (StTP-80AA gi21562 240bp) and eGFP constituted fusion protein, and its localization in tobacco cells was observed by instantaneous expression. Amplified the encoding gene sequence of StTP-80AA and connected to pBI-eGFP, express fusion protein StTP-80AA-eGFP, this vector called pBIL-eGFP. pBIL-eGFP have one multiple enzyme cutting sites (XhoI-SalI-KpnI-HpaI-SmaI) which could detect the effect of signal peptides extending to 80 amino acids. It was also advantageous to insert the detection gene. EcTSR, CmMS and eGFP were fused by StTP-80AA, and their localization in tobacco cells was observed by transient expression. The EcTSR gene and StTP-80AA were connected to the pBI-eGFP construction vector pStTP-80AA-EcTSR-eGFP. The CmMS gene and StTP-80AA were connected to the pBI-eGFP construction vector pStTP-80AA-CmMS-eGFP (more details of plasmid structure - Figure 1).

Building of vectors for protein expression mediated by CTPs with self-cutting site

Amplified PCS1 and PCS2 sequences, were added to vector pBI-eGFP, the pStTp-PCS1-eGFP and pStTp-PCS2-eGFP were constructed. The original signal peptide DNA sequence of pStTP-80AA-EcTSReGFP was replaced by PCS1 and PCS2 sequence, and pStTP-PCS1-EcTSR-eGFP and pStTP-PCS2-EcTSReGFP were constructed (Figure 2 - more details of plasmid structure). See accessory for PCS1 and PCS2 sequence.

Fusion protein instantaneous expression and Western blot analysis

All transient expression vectors that were correctly sequenced were transformed by freezing-thawing method to convert root cancer agrobacterium strain LBA4404. The tobacco aseptic seedling leaves were cut and placed in a Petri dish. The back was dripped into the right amount of bacteria, with a glass syringe suction bacteria liquid penetration blade to wound parent, placed in a Petri dish with a wet filter paper. Covered the Petri dish lid, and avoided light culture for 48h-96h.

Results

80 amino acids of Potato chloroplast localization signal peptides was needed to sufficiently localize EcTSR protein into tobacco chloroplasts 
The 58 amino acids of potato $\mathrm{rbcS}$ (StTP-58AA) contains information for positioning foreign proteins to the tobacco chloroplast, it could locate EcGCL, OsGLO3, EcCAT in our study (Figure 1a-c).

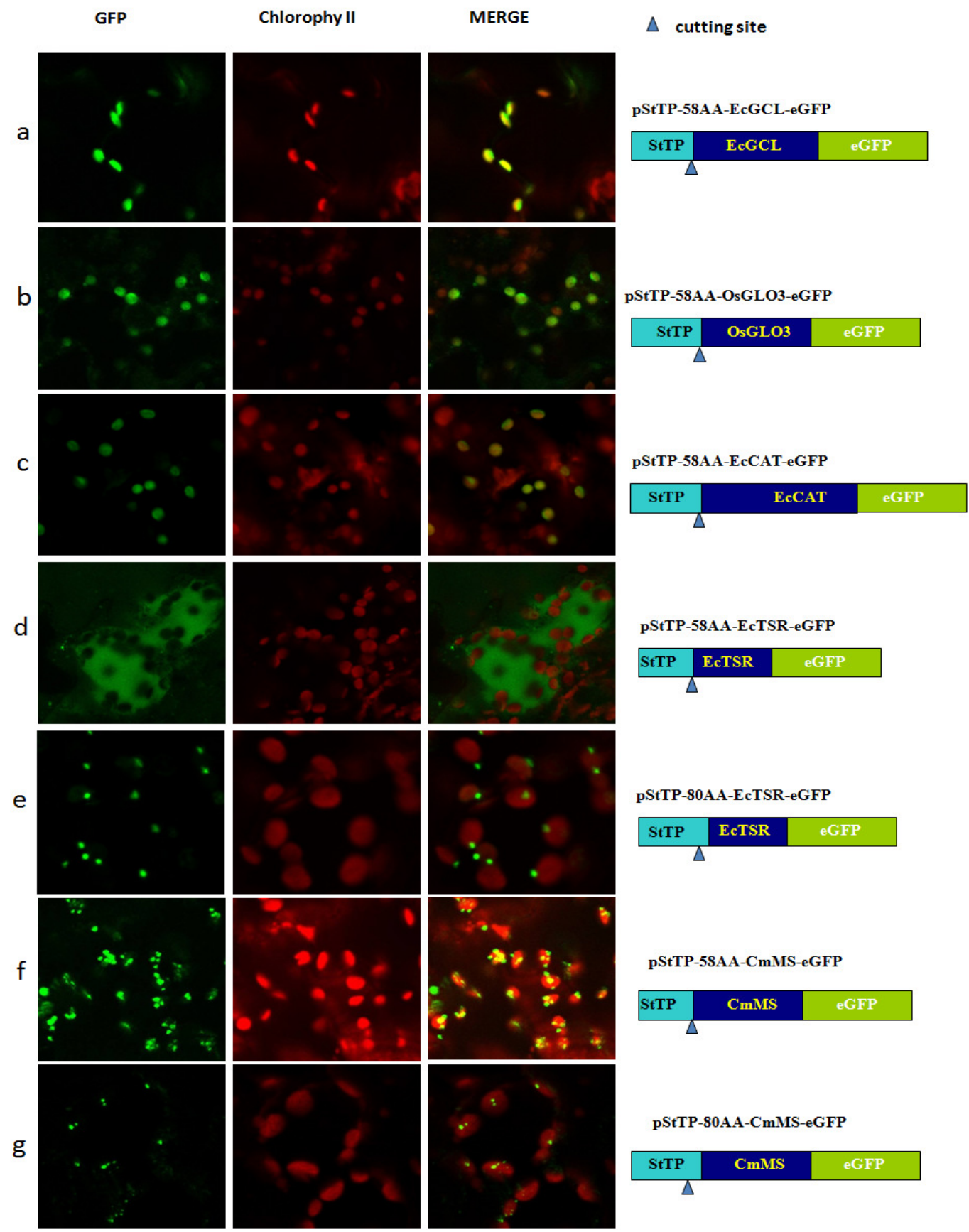

Figure 1. Chloroplast localization of EcGCL, OsGLO3, EcCAT, EcTSR, CmMS mediated by transport peptides of potato $\mathrm{rbcS}$. StTP Chloroplast transport peptides of potato rbcS, OsGLO3 Rice Glycolate oxidase 3, EcGCL E. coli Glyoxylate carboligase, EcCAT E. coli Catalase, EcTSR E. coli hydroxymalonate semi-aldehyde reductase, CmMS cucumber malate synthase 
StTP-58AA could not localize EcTSR into the tobacco chloroplasts, and GFP fluorescence was distributed in the whole cell, and its fluorescence could not overlap with chloroplast auto-fluorescence (Figure 1d) (Shen et al., 2017). Potato rbcS chloroplast positioning signal peptide was extended to 80 amino acids (StTP-80AA), GFP fluorescence crowded around the chloroplast granuli form and suggesting that the EcTSR chloroplast localization is improved (Figure 1e). CmMS protein was fused with 58 amino acids and 80 amino acid chloroplast localization signal peptides respectively, both of them showed that these proteins were clustered around the chloroplasts (Figure 1f-g).

\section{Signal peptide PCS1 can locate protein and self-cut}

PCS1 and PCS2 contain two cutting sites, one cutting site of the potato rbcS signaling peptide was near 58 amino acids, and the other was the additional shearing site. Transient expression indicates that PCS1 and PCS2 was able to well localize EcTSR into tobacco chloroplast, the same as that of the signal peptide of 80 amino acids, all of them located at chloroplast and concentrated in the outer membrane of chloroplast (Figure 2).

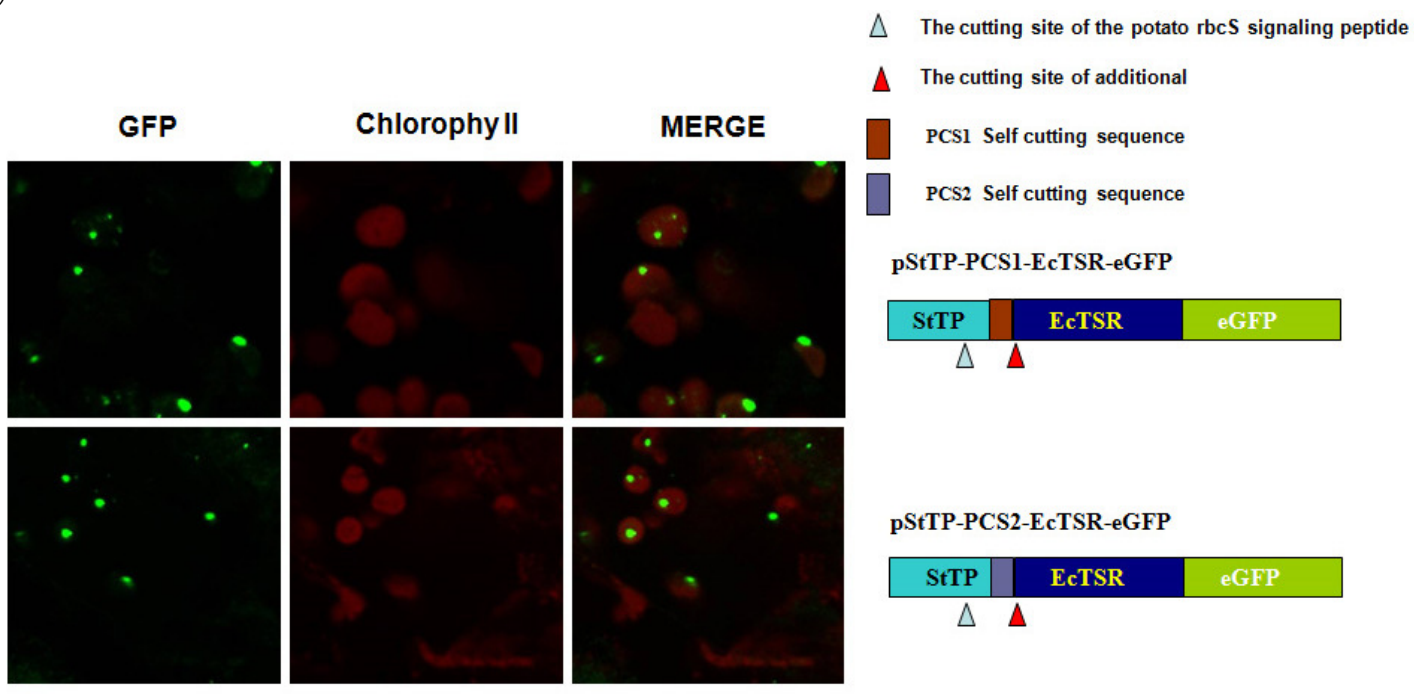

Figure 2. Chloroplast localization of proteins mediated by rbcS transport peptides with Selfcutting sites.StTP chloroplast transport peptides of potato rbcS; EcTSR Tartronate semialdehyde reductase

The protein of transient expression was analyzed, and the pBI-eGFP was used as the reference. The results showed that the 42 amino acids that PCS1 increased compared with StTP-58AA were excised, while the 42 amino acids of PCS2 were not removed (Figure 3a).

The EcTSR protein was mediated by a signal peptide of 58 amino acids. could detect 2 hybridization signals near $60-70 \mathrm{kD}$ (Figure $3 \mathrm{~b}$ ). the second detection band was indicated the signal peptide about $6 \mathrm{kD}$ was removed. The EcTSR protein was mediated by 80 amino acids, only had 1 protein hybridization signal, and the size was the same as the EcTSR-eGFP protein, and the fusion of signal peptide cutting efficiency was improved.

The EcTSR protein was mediated by PCS1, the signal peptide was removed. The EcTSR protein was mediated by PCS2, the signal peptide was not removed. It can be seen from the above results that the selfcutting site introduced in PCS1 completes the cutting, while the self-cutting site of PCS2 fails to complete the cutting. 
a
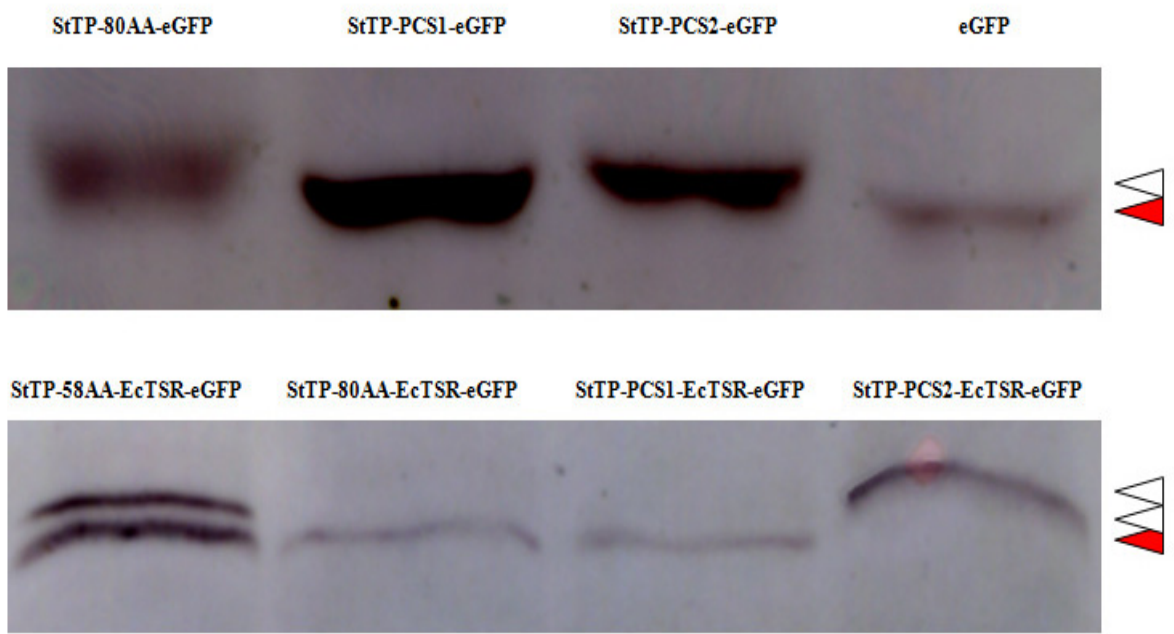

$<$ precursor form $\longrightarrow$ mature form

Figure 3. Western blot of the fusion protein with self-cleavage sites. StTP Chloroplast transport peptides of potato rbcS; EcTSR Tartronate semialdehyde reductase, PCS1 Peptide of selfcleavage site 1, PCS2 Peptide of self-cleavage site 2

\section{Full spectrum scanning analysis confirm PCS1-EcTSR-eGFP enter tobacco chloroplast}

The subcellular localization of fusion protein StTP-PCS1-EcTSR-eGFP in tobacco leaves was analyzed by using the Zeiss 780/7live laser scanning confocal microscope. As shown in Figure 4, three points in a chloroplast were selected to perform full spectrum scanning, the three points were selected from GFP signals the strongest area 1 (red dots), central area of observation 2 (light green) and edge area 3 (dark blue) and they were analyzed with three areas of 400-700 nm spectral data. The results showed that the peaks of 670-690 nm (ChlorophyII) were basically the same, indicating that all three regions were in the chloroplast. In the vicinity of $509 \mathrm{~nm}$ (GFP), area 2 had a high peak value, whose value was weaker than that of region 1 , and the observed edge region 3 had no peak at $509 \mathrm{~nm}$. The results showed that fusion proteins entered the chloroplast, and the GFP fluorescence signal was covered by the GFP fluorescence signal of the fusion protein.

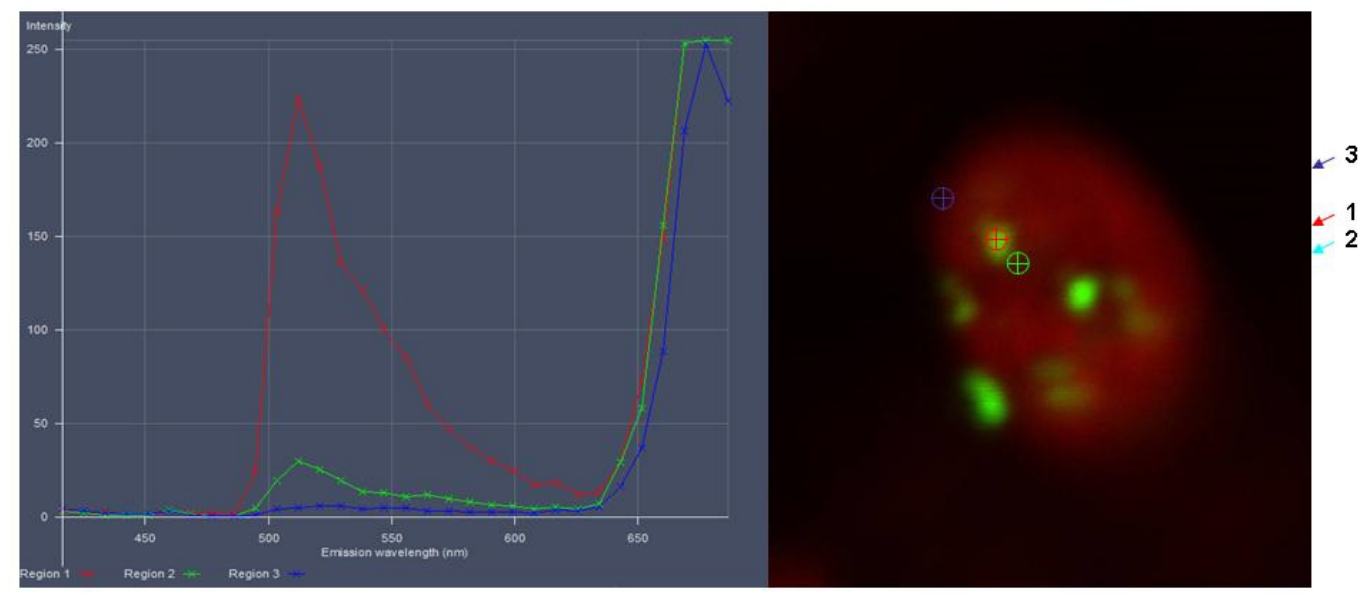

Figure 4. Full spectrum scanning of StTP-PCS1-EcTSR-eGFP fusion protein transient expressed by tobacco leaves 


\section{Discussion}

It is generally believed that plant plasmids that originate from symbiotic cyanobacteria in cells are semiautonomous organelles and they always encode 100-300 genes (Bruce, 2000). While, the rest of more than 3000 genes would be encoded and expressed through nuclear genome and all these kinds of protein were transported to chloroplasts by signal peptides to perform their functions (Bruce, 2000). These genes are first translated into precursor proteins in the cytoplasm and transported to the chloroplasts via their $\mathrm{N}$-terminal chloroplast signal peptide (Jarvis, 2008). Chloroplast transport of precursor proteins are divided into three stages: First these proteins were transported to chloroplast outer membrane through cytokines, next the proteins enter into chloroplast stroma by transmembrane transportation, then signal peptides are removed and folded into mature protein (Lee et al., 2013).

Potato rbcS signal peptides could not localize EcTSR into chloroplasts or had very low localization efficiency. EPSP from Salmonella typhimurium could only be localized to tobacco chloroplasts when 24 amino acids in the mature region of $\mathrm{rbcS}$ were added to pea rbcS signal peptides (Comai et al., 1988). Bionda et al. (2010) reported that chloroplast signaling peptide with a length of more than 60 amino acids (including the sequence behind the cleavage site) are the most suitable. When the target protein is easy to fold tightly, too short signaling peptides may decrease localization efficiency. The possible reason is that the interaction between signaling peptides and transporters (such as Hsp70) requires a certain space (Bionda et al., 2010). According to the literature mentioned above, we extended sequence of the potato rbcS to 80 amino acids in this study. The results proved that the efficiency of localization of EcTSR was significantly improved, which was consistent with the above reports, indicating that the 20 amino acids behind the rbcS cleavage site of potato played a key role in the chloroplast localization of protein EcTSR.

Potato rbcS signal peptide has different localization efficiency for different proteins, and there is no localization, which may be related to the folding speed and strength of the target protein. Similar problems appeared in EcTSR and EcGCL when they located into the chloroplast with the rice rbcS signaling peptide (rCTP). The tertiary structure of EcTSR, rather than the specific amino acid sequence or secondary structure, affects the chloroplast localization function of $\mathrm{rCTP}$, and this effect depends on the degree of proximity between them (Shen et al., 2017). This study suggests that whether the length of the N-terminal unfolded region of exogenous protein or the $\mathrm{rbcS}$ protein itself would affect chloroplast localization in rCTP mediated chloroplast localization. When the length of the unfolded region is less than 20 amino acids, the efficiency of chloroplast localization decreases.

However, in tobacco research, the tertiary structure of EcTSR may also affect the chloroplast localization function of potato $\mathrm{rbcS}$ signal peptide, but the difficulty in locating chloroplasts by EcGCL protein didn't arise. This may be because the localization efficiency depends on both signal peptide and localization protein. This phenomenon deserves further study.

It is noteworthy that after the potato rbcS signal peptide was prolonged, the GFP fluorescence of EcTSR and CmMS which aggregated on the chloroplast outer membrane in granular form could be observed with fluorescence microscopy. In this case, Bionda et al. (2010) and other studies have similar reports, and believe that the protein aggregation in the chloroplast outer membrane may be due to its transmembrane efficiency (Lee et al., 2006; Bionda et al., 2010).

Traditionally, chloroplast proteins remain unfolded with the help of molecular chaperones till they finish transmembrane and folded into functional mature proteins in chloroplasts (Bruce, 2000). Ruprecht $e t$ al. (2010) pointed out that chloroplast proteins might fold immediately after translation. Folded proteins are firstly transported to the outer membrane surface of chloroplast, then restored to their linear unfolded state by molecular chaperones, and then folded again after transmembrane entry into the chloroplast (Ruprecht et al., 2010). According to this view, we believe that the slow recovery of linear state of the CmMS, EcTSR and other proteins may be due to their tight folding when they were transported to the chloroplast outer membrane, thus 
affecting the transmembrane efficiency, then resulting in its aggregation in the chloroplast outer membrane. Full spectrum scanning analysis showed that these proteins could enter chloroplasts.

The second problem of protein chloroplast localization is signal peptide cleavage. Chloroplast transport signal peptides are cleaved by chloroplast matrix processing peptidase SPP, while protein transports to thylakoids require thylakoid processing peptidase cleavage (Teixeira and Glaser, 2013). The cleavage and degradation of signal peptides were studied in detail by Richter and Lamppa (2002). They suggested that the cleavage of signal peptides of iron redox protein precursors was determined by the specific interaction of 12 amino acids at the C-terminal of the signal peptide with SPP (Richter and Lamppa, 2002).

After prolonging the potato signal peptide to 80 amino acids, the chloroplast localization of EcTSR protein was improved, but the $\mathrm{N}$-terminal of mature protein was increased by 20 amino acid residues. The addition of this sequence may affect the enzyme activity and physiological function. After connecting eGFP and EcTSR, the signal peptide PCS1 with cleavage site could locate the protein normally and cleave the signal peptide PCS1. PCS1 can increase the localization efficiency of exogenous proteins, and then completely remove signal peptides.

\section{Acknowledgements}

This work was supported by the project of Youth Fund of Yangtze University, grant number “2015cqn83”.

Accessory

PCS1 DNA sequence

5 -

ATGGCTTCCTCTGTTATTTCCTCTGCAGCTGTTGCTACACGCACCAATGTTACACAAGC TGGCAGCATGATTGCACCTTTCACTGGTCTCAAATCTGCTGCTACTTTCCCTGTTTCAA GGAAGCAAAACCTTGACATCACTTCCATTGCTAGCAATGGTGGAAGAGTTAGGTGCATG CAGGTATGGCCACCAATTAACATGAAGAAGTACGAGACACTCTCATACCTTCCTGATTT GACTATGAGCTCAACCGTCAAGGTCGCCGTCGCCACCCCCAGGATGTCAATCAAGGCC TCCATG-3'

PCS2 DNA sequence

5 '-

ATGGCTTCCTCTGTTATTTCCTCTGCAGCTGTTGCTACACGCACCAATGTTACACAAGC TGGCAGCATGATTGCACCTTTCACTGGTCTCAAATCTGCTGCTACTTTCCCTGTTTCAA GGAAGCAAAACCTTGACATCACTTCCATTGCTAGCAATGGTGGAAGAGTTAGGTGCATG CAGGTATGGCCACCAATTAACATGAAGAAGTACGAGACACTCTCATACCTTCCTGATTT GACTATGAGCTCAGGAAATAGCAGCTTCGGAAATGTGTCCAACGGAGGGAGAATACGAT GTATG-3'

\section{Conflict of Interests}

The authors declare that there are no conflicts of interest related to this article. 


\section{References}

Bionda T, Tillmann B, Simm S, Beilstein K, Ruprecht M, SchleiffE (2010). Chloroplast import signals: the length requirement for translocation in vitro and in vivo. Journal of Molecular Biology 402:510-523.

Bruce BD (2000). Chloroplast transit peptides: structure, function and evolution. Trends in Cell Biology 10(10):440-447.

Bruce BD (2001). The paradox of plastid transit peptides: conservation of function despite divergence in primary structure. Biochimica et Biophysica Acta (BBM)-Molecular Cell Research 1541(1-2):2-21.

Byeon Y, Lee HY, Lee K, Back K (2014). A rice chloroplast transit peptide sequence does not alter the cytoplasmic localization of sheep serotonin N - acetyltransferase expressed in transgenic rice plants. Journal of Pineal Research 57(2):147-157.

Comai L, Larson-Kelly N, Kiser J, Mau CJ, Pokalsky AR, ... Stalker DM (1988). Chloroplast transport of a ribulose bisphosphate carboxylase small subunit-5-enolpyruvyl 3-phosphoshikimate synthase chimeric protein requires part of the mature small subunit in addition to the transit peptide. Journal of Biological Chemistry 263(29):15104-15109.

Corbin DR, Grebenok RJ, Ohnmeiss TE, Greenplate JT, Purcell JP (2001). Expression and chloroplast targeting of cholesterol oxidase in transgenic tobacco plants. Plant Physiology 126:1116-1128.

Dalal J, Lopez H (2015). A photorespiratory bypass increases plant growth and seed yield in biofuel crop Camelina sativa. Biotechnology for Biofuels 8(1): 175 .

Fritz CC, Wolter FP, Schenkemeyer V, Herget T, Schreier PH (1993). The gene family encoding the ribulose-(1,5)bisphosphate carboxylase/oxygenase (Rubisco) small subunit of potato. Gene 137:271-274.

Jarvis P (2008). Targeting of nucleus-encoded proteins to chloroplasts in plants. New Phytologist 179(2):257-285.

Kebeish R, Niessen M, Thiruveedhi K, Bari R, Hirsch HJ, Rosenkranz R, ... Peterhänsel C (2007). Chloroplastic photorespiratory bypass increases photosynthesis and biomass production in Arabidopsis thaliana. Nature Biotechnology 25(5):593-599.

Lee DW, Jung C, Hwang I (2013). Cytosolic events involved in chloroplast protein targeting. Biochimica et Biophysica Acta (BBA)-Molecular Cell Research 1833(2):245-252.

Lee DW, Kim JK, Lee S, Choi S, Kim S, Hwang I (2008). Arabidopsis nuclear-encoded plastid transit peptides contain multiple sequence subgroups with distinctive chloroplast-targeting sequence motifs. The Plant Cell 20(6):1603-1622.

Lee DW, Lee S, Lee GJ, Lee KH, Kim S, Cheong GW, Hwang I (2006). Functional characterization of sequence motifs in the transit peptide of Arabidopsis small subunit of rubisco. Plant Physiology 140:466-483.

Lubben TH, Gatenby AA, Ahlquist P, Keegstra K (1989). Chloroplast import characteristics of chimeric proteins. Plant Molecular Biology 12(1):13-18.

Maier A, Fahnenstich H, von Caemmerer S, Engqvist MK, Weber AP, Flugge UI, Maurino VG (2012). Transgenic introduction of a glycolate oxidative cycle into $A$. thaliana chloroplasts leads to growth improvement. Frontiers in Plant Science 3:38.

Nolke G, Houdelet M, Kreuzaler F, Peterhansel C, Schillberg S (2014). The expression of a recombinant glycolate dehydrogenase polyprotein in potato (Solanum tuberosum) plastids strongly enhances photosynthesis and tuber yield. Plant Biotechnology Journal 12(6):734-742.

Novitskaya L, Trevanion SJ, Driscoll S, Foyer CH, Noctor G (2002). How does photorespiration modulate leaf amino acid contents? A dual approach through modelling and metabolite analysis. Plant Cell \& Environment 25:821-835.

Panstruga R, Hippe-Sanwald S, Lee YK, Lataster M, Lipka V, Fischer R, ... Hirscha HJ (1997). Expression and chloroplasttargeting of active phosphoenolpyruvate synthetase from Escherichia coli in Solanum tuberosum. Plant Science 127:191205.

Peterhansel C, Maurino VG (2011). Photorespiration redesigned. Plant Physiology 155:49-55.

Ratnayake RM, Inoue H, Nonami H, Akita M (2008). Alternative processing of Arabidopsis Hsp70 precursors during protein import into chloroplasts. Bioscience, Biotechnology, and Biochemistry 72(11):2926-1935.

Richter S, Lamppa GK (1998). A chloroplast processing enzyme functions as the general stromal processing peptidase. Proceedings of the National Academy of Science USA 95:7463-7468.

Richter S, Lamppa GK (2002). Determinants for removal and degradation of transit peptides of chloroplast precursor proteins. Journal of Biological Chemistry 277:43888-43894.

Rojas C, Mysore KS (2012). Glycolate oxidase is an alternative source for $\mathrm{H}_{2} \mathrm{O}_{2}$ production during plant defense responses and functions independently from NADPH oxidase. Plant Signaling and Behavior 7(7):752-755. 
Ruprecht M, Bionda T, Sato T, Sommer MS, Endo T, SchleiffE (2010). On the impact of precursor unfolding during protein import into chloroplasts. Molecular Plant 3:499-508.

Shen BR, Zhu CH, Yao Z, Cui LL, Zhang JJ, Yang CW, He ZH, Peng XX (2017). An optimized transit peptide for effective targeting of diverse foreign proteins into chloroplasts in rice. Scientific Reports 7:46231.

Shen BR, Wang LM, Lin XL, Yao Z, Xu HW, Zhu CH, ... Peng XX (2019). Engineering a new chloroplastic photorespiratory bypass to increase photosynthetic efficiency and productivity in rice. Molecular Plant 1-16.

Teixeira PF, Glaser E (2013). Processing peptidases in mitochondria and chloroplasts. Biophysica Acta (BBA)-Molecular Cell Research 1833:360-370.

von Heijne G, Steppuhn J, Herrmann RG (1989). Domain structure of mitochondrial and chloroplast targeting peptides. European Journal of Biochemistry 180:535-545.

Wolter FP, Fritz CC, Willmitzer L, Schell J, Schreier PH (1988). rbcS genes in Solanum tuberosum: conservation of transit peptide and exon shuffling during evolution. Proceedings of the National Academy of Science USA 85:846-850.

Xin CP, Tholen D, Devloo V, Zhu XG (2015). The benefits of photorespiratory bypasses: how can they work? Plant Physiology 167:574-585.

Zhang Z, Xu Y, Xie Z, Li X, He ZH, Peng XX (2016). Association-dissociation of glycolate oxidase with catalase in rice: a potential switch to modulate intracellular $\mathrm{H}_{2} \mathrm{O}_{2}$ levels. Molecular Plant 9:737-748.

OPEN ACCESS

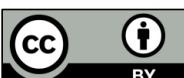

The journal offers free, immediate, and unrestricted access to peer-reviewed research and scholarly work. Users are allowed to read, download, copy, distribute, print, search, or link to the full texts of the articles, or use them for any other lawful purpose, without asking prior permission from the publisher or the author.

License - Papers published in Notulae Botanicae Horti Agrobotanici Cluj-Napoca are Open-Access, distributed under the terms and conditions of the Creative Commons Attribution (CC BY) License.

(c) Articles by the authors; UASVM, Cluj-Napoca, Romania. The journal allows the author(s) to hold the copyright/to retain publishing rights without restriction. 